\title{
Muon diffusion and trapping in chalcopyrite semiconductors
}

\author{
R.C. Vilão ${ }^{\mathrm{a}, *}$, J.M. Gil ${ }^{\mathrm{a}}$, H.V. Alberto ${ }^{\mathrm{a}}$, J. Piroto Duarte ${ }^{\mathrm{a}}$, N. Ayres de Campos ${ }^{\mathrm{a}}$, \\ A. Weidinger ${ }^{\mathrm{b}}$, M.V. Yakusheve ${ }^{\mathrm{c}}$, S.F.J. Cox ${ }^{\mathrm{d}, \mathrm{e}}$ \\ ${ }^{a}$ Physics Department, University of Coimbra, P-3004-516 Coimbra, Portugal \\ ${ }^{\mathrm{b}}$ Hahn-Meitner Institut Berlin, Glienicker Strasse, 100, D-14109 Berlin, Germany \\ ${ }^{\mathrm{c}}$ Department of Physics and Applied Physics, 107 Rottenrow, Strathclyde University, Glasgow G4 ONG, UK \\ ${ }^{\mathrm{d}}$ ISIS Facility, Rutherford Appleton Laboratory, Chilton, Didcot, Oxon OX11 0QX, UK \\ ${ }^{\mathrm{e}}$ Physics Department, University College London, WC1E 6BT, UK
}

\begin{abstract}
The diffusion parameters of diamagnetic muons in chalcopyrites $\mathrm{CuInSe}_{2}, \mathrm{CuInS}_{2}, \mathrm{CuInTe} \mathrm{CuGaTe}_{2}$ and $\left(\mathrm{Ag}_{0.25} \mathrm{Cu}_{0.75}\right) \mathrm{InSe}_{2}$ were obtained by $\mu \mathrm{SR}$ methods. The variations among the different compositions were found to validate the anion-antibonding localization model. The application of a two-state model to the zero-field data revealed muon trapping by defects. The dipolar width at the trap and the number of jumps before trapping were determined. The $\mathrm{Cu}$ vacancy is identified as the trapping center in $\mathrm{CuInSe}_{2}$ and the energy depth of the trap has been determined.

(C) 2002 Elsevier Science B.V. All rights reserved.
\end{abstract}

Keywords: Muon diffusion; Muon trapping; Chalcopyrite semiconductors; Structural defects

Chalcopyrites constitute a very relevant topic in solar-cell research and are already being used in industry [1]. Their structure is derived from the II-VI compounds with zincblende structure, but the cations alternate between groups I and III elements and the unit cell is slightly tetragonally distorted. The most prominent material used so far in solar cells is $\mathrm{CuInSe}_{2}$. In this material the formation probabilities for the most common defects in various stoichiometric cases are known from theoretical calculations performed by Zhang et al. [2].

In previous work $[3,4]$, we have identified the diamagnetic muon site in $\mathrm{CuInSe}_{2}$ at low temperatures as being the Se anion anti-bonding site, considering both the transverse-field depolarisa-

\footnotetext{
*Corresponding author. Fax: + 351-239-829158.

E-mail address: ruivilao@ci.uc.pt (R.C. Vilão).
}

tion rate and the zero-field dipolar width. We have also studied the muon site and diffusion in $\mathrm{CuInSe}_{2}$ and $\mathrm{CuInS}_{2}$. In this work we extend the study to $\mathrm{CuInTe} \mathrm{I}_{2}$ (completing the systematics over the group VI anions). We also extend this research to $\mathrm{CuGaTe}_{2}$ and to the mixed quaternary compound $\left(\mathrm{Ag}_{0.25} \mathrm{Cu}_{0.75}\right) \mathrm{InSe}_{2}$. A two-state model analysis of the observed muon trapping in structural defects is also presented.

The CuInTe $2, \mathrm{CuGaTe}_{2}$ and $\left(\mathrm{Ag}_{0.25} \mathrm{Cu}_{0.75}\right) \mathrm{InSe}_{2}$ samples used in this work were single crystals grown by the vertical Bridgman technique. The $\mathrm{CuInSe}_{2}$ p- and n-type single crystals and the $\mathrm{CuInS}_{2}$ crystallites were samples used previously $[3,4]$. Transverse field experiments were performed at the Paul Scherrer Institut (GPS instrument), Switzerland, at temperatures from 2 to $312 \mathrm{~K}$. Zero-field experiments took place at the ISIS Facility, Rutherford-Appleton Laboratory, UK, 
in the temperature range from 10 to $500 \mathrm{~K}$. It was found, by transverse-field measurements, that the majority of the implanted muons were in a diamagnetic state for all the samples studied, as had been already observed for $\mathrm{CuInSe}_{2}$ and $\mathrm{CuInS}_{2}$ [3]. At low temperatures a small paramagnetic fraction of implanted muons (5-10\%) was found, this fraction converting to diamagnetic around $100 \mathrm{~K}$ in all compounds studied [3].

A preliminary qualitative description of the behaviour of the diamagnetic muon was obtained by fitting the zero-field data with a singlecomponent static Kubo-Toyabe (KT) function [5]. Fig. 1 shows the values of dipolar width $\Delta$ obtained for some of the compounds. The other compounds studied show similar results. This function only fits the data well in the lower temperature range and in the vicinity of the higher temperature range peak. Although the value of $\Delta$ obtained has no direct physical meaning in the other temperature ranges, this preliminary analysis serves the purpose of distinguishing the different ranges and suggesting appropriate physical interpretation and analysis. We can thus distinguish three main ranges in Fig. 1: low-temperature; the range of sharp decrease in $\Delta$; the higher temperature range, where $\Delta$ is either stable or shows a peak. Each of these ranges suggest a different mechanism and fitting procedure, which will be discussed in the following paragraphs.

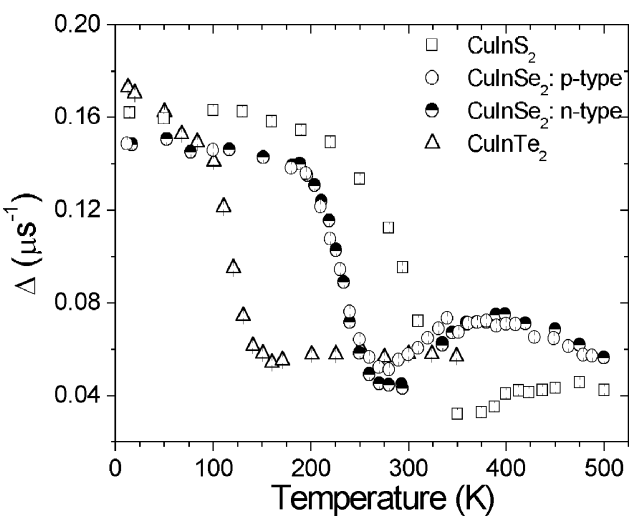

Fig. 1. Temperature dependence of the zero-field dipolar width $\Delta$, measured on $\mathrm{CuInSe}_{2}$ and $\mathrm{CuInTe}_{2}$ single crystals and on $\mathrm{CuInS}_{2}$ crystallites. The $\Delta$ values were obtained from fits of a single-component static KT function.
At the lower temperatures (Fig. 1), the muon is static in the lattice, with $\Delta$ characteristic of the occupied site, the results obtained for the newly measured samples being compatible with localisation at the anion anti-bonding site [3].

The sharp decrease of the one-component fitted $\Delta$ with increasing temperature (Fig. 1) results from diffusion of the muon. Jump rates as a function of temperature were obtained from more appropriate fits using a dynamic KT function. Fig. 2 shows Arrhenius plots of the jump rates for some of the samples. Activation energies and pre-exponential factors for all samples were obtained by linear fits to Arrhenius plots and are presented in Table 1, together with the limits of the temperature range where diffusion is observed. These limits differ among the compounds only when a different anion is present, being the highest for $\mathrm{S}$ and lowest for Te. This reflects the decrease in the strength of the muon-chalcogen bond from $\mathrm{S}$ to Te. Concomitantly, we observe the highest activation energy for the $\mathrm{S}$ chalcopyrite and the lowest for the Te chalcopyrites.

In the higher temperature range of Fig. 1 the decrease of $\Delta$ ceases and its value either stabilises, as in $\mathrm{CuInTe}_{2}$, or shows a peak at some temperature value, as in $\mathrm{CuInSe}_{2}, \mathrm{CuInS}_{2}, \mathrm{Cu}$ $\mathrm{GaTe}_{2}$ and $\left(\mathrm{Ag}_{0.25} \mathrm{Cu}_{0.75}\right) \mathrm{InSe}_{2}$. This is interpreted

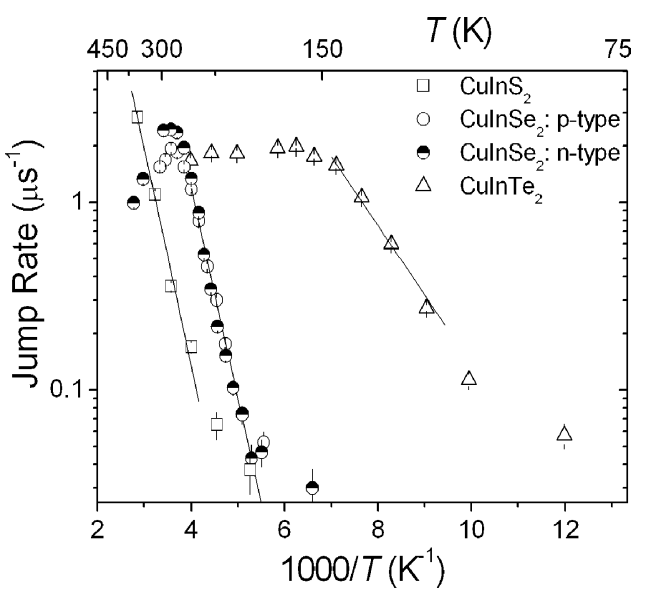

Fig. 2. The jump rate as a function of temperature for diamagnetic muons diffusing in $\mathrm{CuInS}_{2}, \mathrm{CuInSe}_{2}$ and $\mathrm{CuInTe} \mathrm{I}_{2}$. The jump rate values were obtained by fitting a dynamic KT function to the zero-field data. The lines are Arrhenius fits to these plots (Table 1). 
as an interruption of the diffusion process by trapping in the vicinity of defects. We assume that in this temperature range the polarisation of diffusing muons is described by an exponential relaxation $\lambda$, which is the high jump-rate limit of the dynamic KT function. The polarisation of trapped muons is described by a static KT function $\left(\mathrm{KT}_{\text {stat }}\right)$ with a $\Delta_{\text {trap }}$ characteristic of the trapping site. For rapidly diffusing muons that are trapped after a mean 'free' time $\tau_{\mathrm{f}}$ the zero-field polarisation is described (assuming that the muon stays in the trap) by the function:

$$
\begin{aligned}
P(t)= & \exp \left(-\frac{t}{\tau_{\mathrm{f}}}\right) \exp (-\lambda t) \\
& +\frac{1}{\tau_{\mathrm{f}}} \int_{0}^{t} \mathrm{KT}_{\text {stat }}\left(t-t^{\prime}\right) \exp \left(-\frac{t^{\prime}}{\tau_{\mathrm{f}}}\right) \\
& \times \exp \left(-\lambda t^{\prime}\right) \mathrm{d} t^{\prime}
\end{aligned}
$$

The inverse free time is proportional to the trapping radius $r_{\text {trap }}$, the concentration of traps $c_{\text {trap }}$ and the muon's diffusion constant $D_{\mu}[6]$ :

$\frac{1}{\tau_{\mathrm{f}}}=4 \pi r_{\text {trap }} c_{\text {trap }} D_{\mu}$

Table 1

Temperature range, activation energies and pre-exponential factors for muon diffusion in each chalcopyrite

\begin{tabular}{llll}
\hline & $\begin{array}{l}\text { Diffusion } \\
\text { range (K) }\end{array}$ & $E_{\mathrm{a}}(\mathrm{meV})$ & $v_{0}\left(10^{9} \mathrm{~s}^{-1}\right)$ \\
\hline $\mathrm{CuInS}_{2}$ & $250-300$ & 225 & 5 \\
$\mathrm{CuInSe}$ & $200-250$ & 220 & 34 \\
$\left(\mathrm{Ag}_{0.25} \mathrm{Cu}_{0.75}\right) \mathrm{InSe}_{2}$ & $200-250$ & 184 & 5 \\
$\mathrm{CuInTe}_{2}$ & $100-150$ & 73 & 0.7 \\
$\mathrm{CuGaTe}_{2}$ & $100-150$ & 56 & 0.2 \\
\hline
\end{tabular}

where $D_{\mu}$ is proportional to the muon jump rate $v_{\text {jump }}$ [6]. Thus the inverse free time is simply proportional to the muon jump rate and is expected to follow an Arrhenius law. We have performed a simultaneous fit to all the zero-field data where trapping is the dominant feature, by imposing Arrhenius behaviour on the individual free times with the activation energies of Table 1 . The corresponding temperature ranges, as well as the relevant fitting parameters, are summarised in Table 2. The mean number of jumps $N$ before trapping $\left(N=v_{0} \tau_{0 \mathrm{f}}\right)$ is also indicated.

We shall now focus on the $\mathrm{CuInSe} \mathrm{S}_{2}$ results. The low value of the dipolar width $\Delta_{\text {trap }}$ at the trap (the same for both the $\mathrm{n}$ - and the p-type samples) indicates that the muon is much further apart from spin-carrying nuclei than at low temperatures. A vacancy is therefore likely to be the defect responsible for the trapping. Dipolar width simulations for the most probable vacancies in our samples indicate $\Delta_{\mathrm{I}}=0.0997 \mu \mathrm{s}^{-1}$ for $\mathrm{Cu}$ vacancies and $\Delta_{\mathrm{III}}=0.085 \mu \mathrm{s}^{-1}$ for In vacancies, as shown in Table 2. Local distortions around the muon (not considered in the simulations) tend to reduce $\Delta$ (10-20\% reduction being usual [7]), which makes the value for $\mathrm{Cu}$ vacancies consistent with our experimental result. Since the formation enthalpy of $\mathrm{Cu}$ vacancies is lower than that of In vacancies [2] it is expected that the $\mathrm{Cu}$ vacancies are more abundant. We thus identify the $\mathrm{Cu}$ vacancies as the defect responsible for muon trapping in $\mathrm{CuInSe}_{2}$. It is not possible to determine the defect concentrations, since the trapping radius is unknown. Assuming the trapping radius to be equal to the jump step (about $2 \AA$ ), we obtain concentrations on the order of $10^{20} \mathrm{~cm}^{-3}$, which

Table 2

Temperature range, dipolar width at the trapping site $\Delta_{\text {trap }}$ and pre-exponential factor $\tau_{0}$ (from fits), corresponding mean number $N$ of

\begin{tabular}{|c|c|c|c|c|c|c|}
\hline & Trapping range $(\mathrm{K})$ & $\tau_{0}\left(10^{-4} \mu \mathrm{s}\right)$ & $\Delta_{\text {trap }}\left(\mu \mathrm{s}^{-1}\right)$ & $\Delta_{\mathrm{I}}\left(\mu \mathrm{s}^{-1}\right)$ & $\Delta_{\mathrm{III}}\left(\mu \mathrm{s}^{-1}\right)$ & $N$ \\
\hline $\mathrm{CuInS}_{2}$ & $350-400$ & $13(5)$ & $0.049(3)$ & 0.1140 & 0.097 & 7 \\
\hline $\mathrm{CuInSe}_{2}$ (n-type) & $260-340$ & $10.3(27)$ & $0.082(5)$ & 0.0997 & 0.085 & 35 \\
\hline $\mathrm{CuInSe}_{2}$ (p-type) & $260-340$ & $7.2(4)$ & $0.084(5)$ & 0.0997 & 0.085 & 25 \\
\hline$\left(\mathrm{Ag}_{0.25} \mathrm{Cu}_{0.75}\right) \mathrm{InSe}_{2}$ & $300-390$ & $3.9(9)$ & $0.098(9)$ & 0.1041 & 0.0894 & 2 \\
\hline $\mathrm{CuGaTe}_{2}$ & $140-400$ & $24570(2690)$ & $0.165(15)$ & 0.0473 & 0.0484 & 491 \\
\hline
\end{tabular}
jumps before trapping, and calculated dipolar widths at group-I and group-III cation vacancies $\left(\Delta_{\mathrm{I}}\right.$ and $\Delta_{\mathrm{III}}$, respectively), for each sample 
seems too high, even for these highly doped samples. The trapping radius for the muon must therefore be much higher, as expected for negatively charged $\mathrm{Cu}$ vacancies, whose action on the rapidly diffusing muon is extended probably over many unit cells.

Regarding our experimental results on the other chalcopyrites, we may point out that:

(1) The dipolar widths measured in $\mathrm{CuInS}_{2}$ and $\left(\mathrm{Ag}_{0.25} \mathrm{Cu}_{0.75}\right) \mathrm{InSe}_{2}$ are compatible with trapping at vacancies, although for $\mathrm{CuInS}_{2}$ this kind of trapping seems to demand an exceptionally large local distortion.

(2) In $\mathrm{CuGaTe}_{2}$ a broad trapping peak is also observed, but the fitting results are clearly incompatible with trapping at vacancies. The high dipolar width observed is probably related to the trapping at interstitial sites close to the $\mathrm{Ga}$ or $\mathrm{Cu}$ which have spincarrying nuclei.

(3) $\mathrm{CuInTe}_{2}$ is a special case as it shows no trapping peak. However, the dipolar width $\Delta$ is not seen to decrease towards zero as expected for simple diffusion, rather flattening around $\Delta=0.06 \mu \mathrm{s}^{-1}$ for temperatures above $150 \mathrm{~K}$. This suggests that the muon is being trapped and detrapped very rapidly, the trapping peak being consequently smoothed.

Detrapping of the muon is expected to become probable when the thermal energy is of the order of $E_{\text {trap }}$, therefore reducing again the single component $\Delta$ through diffusion. Such a reduction is observed for CuInSe 2 above $375 \mathrm{~K}$ (Fig. 1). If the muon remains trapped for a mean time $\tau_{t}$ much larger than the muon lifetime, we may model the muons' behaviour by modifying Eq. (1) in order to allow detrapping:

$$
\begin{aligned}
P(t)= & \exp \left(-\frac{t}{\tau_{\mathrm{f}}}\right) \exp (-\lambda t) \\
& +\int_{0}^{t} \exp \left(-\frac{t-t^{\prime}}{\tau_{\mathrm{t}}}\right) \mathrm{KT}_{\text {stat }}\left(t-t^{\prime}\right) \\
& \times \frac{1}{\tau_{\mathrm{f}}} \exp \left(-\frac{t^{\prime}}{\tau_{\mathrm{f}}}\right) \exp \left(-\lambda t^{\prime}\right) \mathrm{d} t^{\prime}
\end{aligned}
$$

$$
\begin{aligned}
& +\int_{0}^{t} \exp \left[-\lambda\left(t-t^{\prime}\right)\right] \\
& \times \frac{1}{\tau_{t}}\left\{\int_{0}^{t^{\prime}} \exp \left(-\frac{t^{\prime}-t^{\prime \prime}}{\tau_{t}}\right) \mathrm{KT}_{\mathrm{stat}}\left(t^{\prime}-t^{\prime \prime}\right)\right. \\
& \left.\times \frac{1}{\tau_{\mathrm{f}}} \exp \left(-\frac{t^{\prime \prime}}{\tau_{\mathrm{f}}}\right) \exp \left(-\lambda t^{\prime \prime}\right) \mathrm{d} t^{\prime \prime}\right\} \mathrm{d} t^{\prime} .
\end{aligned}
$$

Of course, if the process turns out to be too fast, Eq. (3) must be modified in order to accommodate trapping-detrapping succession $[8,9]$.

We have fitted our $\mathrm{CuInSe}_{2}$ data with this model, in order to estimate $E_{\text {trap }}$. Detrapping being a thermally activated process, $\tau_{t}$ is expected to follow an Arrhenius law. As for the trapping, we have performed a simultaneous fit to all relevant data. We have obtained $E_{\text {trap }}=267(40) \mathrm{meV}$ for the p-type sample and $E_{\text {trap }}=664(79) \mathrm{meV}$ for the n-type sample. The pre-exponential factors are of the order of $10^{-2} \mu \mathrm{s}$.

The help of the $\mu$ SR groups at PSI and ISIS in supporting the experiments is gratefully acknowledged. This work was partially supported by FCT and FEDER (Portugal, POCTI/1999/FIS/ 35334), EU TMR program for Large-Scale Facilities (research at ISIS) and EPSRC grant GR/ R74116.

\section{References}

[1] M.A. Green, et al., Prog. Photovoltaics: Res. Appl. 4 (1996) 59.

[2] S.B. Zhang, et al., Phys. Rev. B 57 (1998) 9642.

[3] J.M. Gil, et al., Phys. Rev. B 59 (1999) 1912.

[4] J.M. Gil, et al., Physica B 289-290 (2000) 567.

[5] R.S. Hayano, et al., Phys. Rev. B 20 (1979) 850.

[6] E.B. Karlsson, Solid State Phenomena, Oxford Science Publications, Oxford, 1995.

[7] A. Schenck, Muon Spin Rotation Spectroscopy, Adam Hilger Ltd., Bristol, 1985, p. 60ff.

[8] M. Borghini, et al., Phys. Rev. Lett. 40 (1978) 1723.

[9] R. Hempelmann, et al., Solid State Ionics 107 (1998) 269. 